\title{
Feasibility of the evidence-based cognitive telerehabilitation program Remind for patients with primary brain tumors
}

\author{
Sophie D. van der Linden ${ }^{1,2} \cdot$ Margriet M. Sitskoorn ${ }^{2} \cdot$ Geert-Jan M. Rutten $^{1} \cdot$ Karin Gehring $^{1,2}$
}

Received: 8 September 2017 / Accepted: 29 December 2017 / Published online: 10 January 2018

(c) The Author(s) 2018. This article is an open access publication

\begin{abstract}
Many patients with primary brain tumors experience cognitive deficits. Cognitive rehabilitation programs focus on alleviating these deficits, but availability of such programs is limited. Our large randomized controlled trial (RCT) demonstrated positive effects of the cognitive rehabilitation program developed by our group. We converted the program into the iPad-based cognitive rehabilitation program ReMind, to increase its accessibility. The app incorporates psychoeducation, strategy training and retraining. This pilot study in patients with primary brain tumors evaluates the feasibility of the use of the ReMind-app in a clinical (research) setting in terms of accrual, attrition, adherence and patient satisfaction. The intervention commenced 3 months after resective surgery and patients were advised to spend $3 \mathrm{~h}$ per week on the program for 10 weeks. Of 28 eligible patients, 15 patients with presumed low-grade glioma or meningioma provided informed consent. Most important reason for decline was that patients (7) experienced no cognitive complaints. Participants completed on average $71 \%$ of the strategy training and $76 \%$ of the retraining. Some patients evaluated the retraining as too easy. Overall, $85 \%$ of the patients evaluated the intervention as "good" or "excellent". All patients indicated that they would recommend the program to other patients with brain tumors. The ReMind-app is the first evidence-based cognitive telerehabilitation program for adult patients with brain tumors and this pilot study suggests that postoperative cognitive rehabilitation via this app is feasible. Based on patients' feedback, we have expanded the retraining with more difficult exercises. We will evaluate the efficacy of ReMind in an RCT.
\end{abstract}

Keywords Cognitive rehabilitation $\cdot$ Telerehabilitation $\cdot$ eHealth $\cdot$ Glioma $\cdot$ Meningioma $\cdot$ Neurosurgery

\section{Introduction}

Many patients with primary brain tumors suffer from cognitive deficits $[1,2]$. These deficits can cause difficulties in patients' everyday lives and affect their quality of life [3, 4]. Reported prevalence rates of cognitive deficits vary widely, which is partly due to the differences in used methods, but range between 19 and 90\% [2, 5, 6]. Since survival rates are increasing $[7,8]$ and patients are living longer with possible cognitive deficits, management of cognitive deficits becomes an increasingly important part of total care in patients with primary brain tumors.

Sophie D. van der Linden

s.d.vdrlinden@uvt.nl; s.vanderlinden@etz.nl

1 Department of Neurosurgery, Elisabeth-TweeSteden Hospital, Tilburg, The Netherlands

2 Department of Cognitive Neuropsychology, Tilburg University, P.O. Box 90153, 5000 LE Tilburg, The Netherlands
Unfortunately, treatment options for these cognitive deficits are scarce. Over the last years, a few intervention studies have been conducted in brain tumor patients, which demonstrated positive effects of cognitive rehabilitation [9-11]. Our randomized controlled trial (RCT) in 140 glioma patients with stable disease demonstrated positive effects of a 6-week face-to-face cognitive rehabilitation program that consisted of psychoeducation, teaching of use of compensatory skills and retraining $[9,12]$. Despite the positive findings of previous studies and patients' needs for rehabilitation services, cognitive rehabilitation is not always accessible for every patient in clinical practice $[13,14]$. Conventional inperson cognitive rehabilitation can be demanding and costly, due to, amongst others, multiple hospital visits and lengthy face-to-face sessions with professionals.

To overcome some of the limitations of conventional cognitive rehabilitation, a number of studies explored the possibilities of cognitive telerehabilitation programs in other neurological and oncological patient populations [15-18]. Cognitive telerehabilitation is a form of eHealth, and it 
is defined as "the use of information and communication technologies to provide rehabilitation services to people remotely in their homes or other environments" [19]. To our knowledge, no studies on cognitive telerehabilitation have been conducted in adult patients with brain tumors.

Based on the positive findings of our previous RCT, and ongoing requests of doctors and patients to utilize the cognitive rehabilitation program, we converted our program into an iPad-based cognitive rehabilitation application, named ReMind. The goal of the development of the ReMind-app was to increase the accessibility of the program to brain tumor patients in a cost-efficient mode of delivery, while maintaining the contents of the original program. Before we initiated an RCT to evaluate the efficacy of ReMind, we conducted a small-scale study to investigate the feasibility of, and potential barriers to, the use of the program in the clinical (research) setting in terms of accrual, attrition, adherence and patient satisfaction. Since cognitive telerehabilitation has not yet been investigated in adult patients with primary brain tumors (i.e. vulnerable patients with higher levels of fatigue, psychological distress and concentration problems), this feasibility study is an important first step.

\section{Methods}

\section{Participants}

Patients with a radiologically suspected supratentorial low-grade glioma or meningioma, who were scheduled for resective surgery in the Elisabeth-TweeSteden Hospital Tilburg, were invited to participate. Patients who met any of the following criteria were excluded: history of intracranial neurosurgery, history of severe psychiatric or neurological disorder, diagnosis of multiple meningioma, complete unfamiliarity with the use of computers, lack of basic proficiency in Dutch, inability to undergo neuropsychological assessment due to motor/language/visual problems, Karnofsky performance score (KPS) below 70 or a premorbid IQ below 85. Patients who were referred to in- or outpatient cognitive rehabilitation were excluded as well. The projected sample size was 15 . Informed consent was obtained from all individual participants included in the study.

\section{Design and procedure}

This single-arm pilot study was approved by the local medical ethical review board (METC Brabant: NL51152.028.14), registered in The Netherlands National Trial Register (NTR 5392).

Two weeks before surgery, patients were informed about the study by a nurse practitioner. Interested patients received an information letter. One day before surgery, patients were hospitalized and neuropsychological assessment (T0) was carried out as part of usual clinical care. At the beginning of the assessment, patients who were willing to participate in the study provided written informed consent. If patients chose to make use of the possibility to involve a significant other in the cognitive rehabilitation trajectory (see below), the significant other had to give informed consent as well. Three months after surgery, a second usual care neuropsychological assessment (T3) was conducted. Immediately afterwards, the cognitive telerehabilitation program ReMind commenced. Three months later (i.e. 6 months after surgery), after completing ReMind, the final neuropsychological assessment took place (T6) for the purpose of this study. Additionally, study-specific evaluation questionnaires were completed. The current study was embedded in standard clinical care provided by the hospital.

\section{Intervention}

\section{The program}

The cognitive telerehabilitation program Remind was developed in a joint patient/researcher initiative and is based on our previously evaluated face-to-face cognitive rehabilitation program $[9,12,20]$. The ReMind-app is provided via an iPad (Fig. 1a) and is available in both Dutch and English. In the current study, the Dutch version was used.

Similar to the original program [12], ReMind consists of compensation training, including psychoeducation and teaching of compensatory skills, and attention retraining (see Fig. 1b, c). In the compensation training, psychoeducation about cognitive functions is provided in six modules, namely on (1) Cognitive functions, (2) Influences, (3) Compensation, (4) Attention, (5) Planning \& control, and (6) Memory. Additionally, in each module, compensatory strategies are taught and many exercises are included to learn to apply these strategies in everyday life. Patients learn, for example, to minimize distraction and deal with time pressure, and to optimally use external devices for support. Due to the strong interdependence of all cognitive functions, the strategy training was designed so that patients should go through all the six modules one by one, to benefit the most from the strategy training. Progression through each module is visualized, with checkmarks at the bottom of the screen (Fig. 1b).

In the retraining part, named $\mathrm{C}$-Car, four different modes of attention are trained, namely sustained, selective, alternating and divided attention. It includes visual and auditory exercises, wherein both verbal and numeric stimuli are presented. All patients started with the same version of this training, independently of their pre-intervention neuropsychological scores. Series of hierarchically graded tasks were used, so that higher levels are reached, if previous levels 

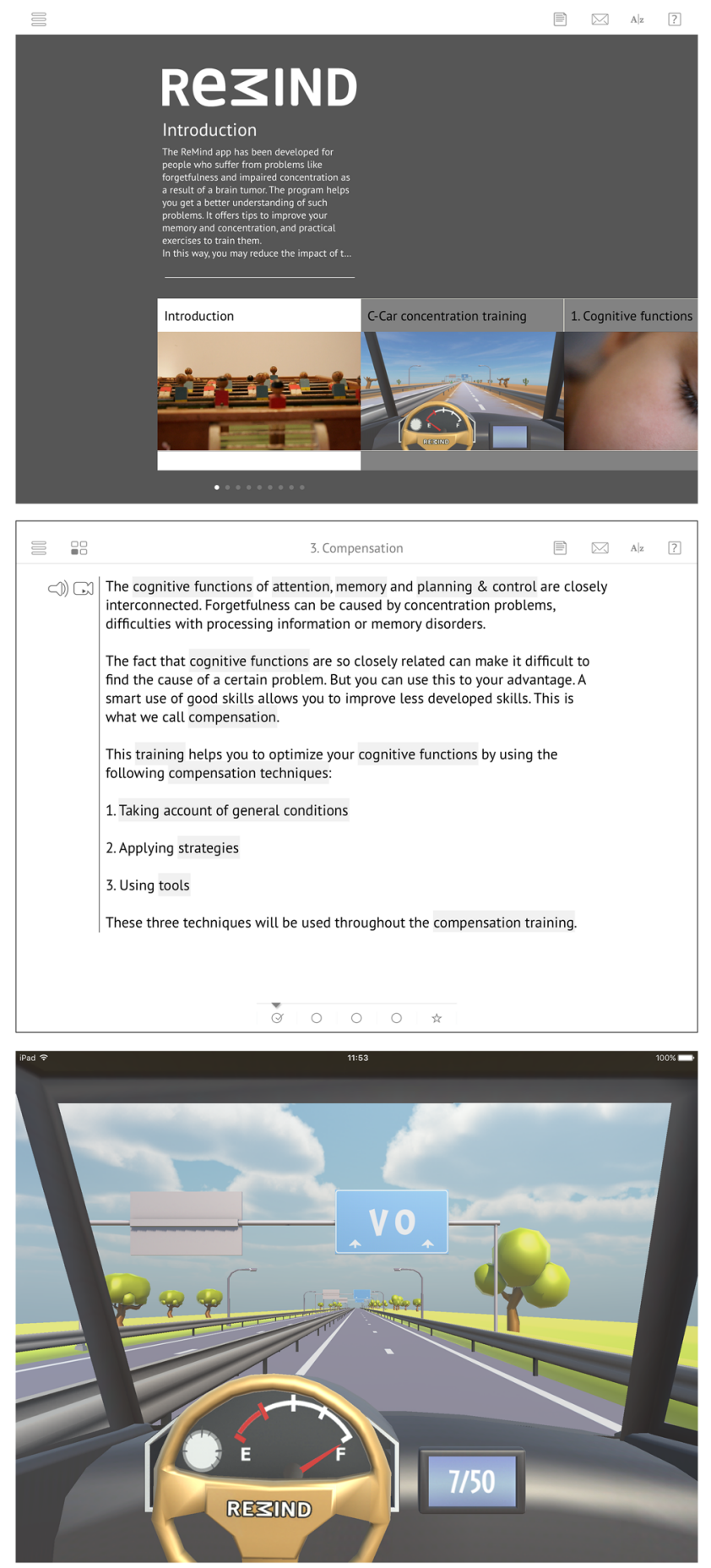

Fig. 1 Screenshots of different parts of ReMind: a homepage, b strategy training, and $\mathbf{c}$ retraining

are mastered. In this manner, the retraining is tailored to the level of the patient. After each exercise, patients receive feedback on their performance.

During the development of the app, optimum use was made of the additional (technical) possibilities the new environment offered. The instructional texts of the strategy training are provided in videos, audio clips and read-only formats and patients can look back as often as they feel necessary. ReMind incorporates several other functions to make it as user-friendly as possible, such as help-overlay screens and links to explanations of important definitions. The program also offers the possibility to involve a significant other, which can be a spouse, family member, friend or professional: the ReMinder. Patients can send this ReMinder an email from anywhere in the program, for example to ask for advice when they get stuck in a text or an exercise.

\section{Guidance}

Three months after surgery, immediately after the second neuropsychological assessment (T3), a face-to-face appointment was planned, to hand over the iPad on which the ReMind-app was installed together with an explanation of the app. During the intervention period, the researcher contacted the patients by telephone every 2 weeks, to check on their progress, plan the course of their training and to address questions. It was advised and expected that patients spent $3 \mathrm{~h}$ per week on the program, to complete the program within 10 weeks. A second face-to-face appointment took place at the end of the program, to retrieve the iPad and to collect the completed questionnaires.

\section{Measures}

\section{Accrual and attrition}

Accrual was defined as the total number of included patients as compared to the number of invited patients. The number of patients who declined participation and reasons for decline were carefully recorded. The same was done for the number of patients who dropped out of the study and reason(s) for this attrition.

\section{Adherence}

Adherence to the program was indicated by both the number of completed module sections in the strategy training and the number of exercises performed in the retraining, each expressed in percentages of total available sections and exercises, respectively. If patients completed $\geq 80 \%$ of both the strategy training and the retraining, adherence was considered acceptable. To calculate mean percentages for the group, a maximum of $100 \%$ per individual was used, even if patients worked through the program more than once. Reasons for non-adherence as reported in the telephone calls during the intervention and in the face-to-face appointment at the end of the program were recorded. 


\section{Patient experience}

After completing the program, patients were requested to fill out a study-specific questionnaire, evaluating their experiences with ReMind (e.g., satisfaction, enjoyment, usefulness and burden), whether they would recommend any changes in (elements of) the program, and if they would recommend it to other patients.

\section{Feasibility of neuropsychological assessments}

Neuropsychological tests and patient-reported outcome measures (PROMs) were administered to describe baseline functioning of the patients and to test the feasibility of procedures for later use on a larger scale. Objective cognitive functioning was assessed by the computerized neuropsychological test battery CNS Vital Signs [21, 22] and three paper-and-pencil tests, namely Letter Fluency, Digit Span (WAIS), and Paired Associates (WMS) [23-25]. Z-scores were calculated using normative data and $Z$-scores $\leq-1.5$ were considered as low. Subjective cognitive functioning was assessed with the Cognitive Failures Questionnaire (CFQ) [26]. Based on Dutch representative normative data [27], a total score of $\geq 42$ was considered as clinically high. Symptoms of anxiety and depression were assessed with the Hospital Anxiety and Depression Scale (HADS) [28, 29], with a cut-off for both scales of $\geq 8$.

\section{Data analysis}

Percentages of eligible, included, excluded and dropped-out patients were calculated. Descriptive statistics of participants are presented. This feasibility study $(n=15)$ was not designed, and therefore not powered, to evaluate the efficacy of ReMind.

\section{Results}

\section{Accrual and attrition}

Data on accrual and attrition are presented in a flow diagram (Fig. 2). Out of 65 consecutive patients who were scheduled to undergo surgery for presumed low-grade glioma/meningioma, 37 patients (57\%) were excluded. Of the 28 eligible patients who were invited to participate, 15 patients provided informed consent (54\%) and 13 patients (46\%) declined. The most important reason for decline was that patients $(n=7)$ did not experience cognitive deficits and felt no need to follow a cognitive rehabilitation program at this stage.

In the 3 months prior to the start of the intervention, one patient withdrew from the study because of lack of cognitive complaints. An additional patient was excluded after informed consent, since she was referred to cognitive rehabilitation elsewhere. No dropout occurred during the intervention phase. Nine participants $(69 \%)$ chose to involve a significant other (in all cases, a spouse).

\section{Demographic, clinical and neuropsychological characteristics}

Table 1 shows the demographic, clinical and neuropsychological characteristics of the sample. Thirteen patients $(38 \%$ female), with a mean age of 52 years (range 40-68), followed the cognitive rehabilitation program and completed all assessments. Six patients were diagnosed with a grade I meningioma, one patient with a WHO grade II meningioma and four patients with a WHO grade II glioma. For two other patients, the radiologically suspected diagnosis of low-grade glioma was not confirmed after surgery (Table 1). After surgery, five patients (38\%) were treated with radiotherapy and three of these patients also received chemotherapy at T3. The majority (69\%) of the patients were highly educated. Before the start of the intervention, seven patients demonstrated low $Z$-scores $(\leq-1.5)$ on one or more measures of objective cognitive functioning. Based on the scores of the HADS, three of the patients possibly suffered from depression, and one of them possibly from anxiety as well (Table 1).

\section{Adherence}

Adherence per participant is presented in Table 1. On average, participants completed $71 \%$ of the strategy training $76 \%$ of the retraining. According to our definition of adherence (completion of $\geq 80 \%$ of both the strategy training and the retraining), seven out of 13 patients (54\%) adhered to the program. Six patients (46\%) completed the entire strategy training and seven patients (54\%) completed the entire retraining. Four patients (31\%) fully completed both the strategy- and retraining. Three participants reported specific circumstances that explained non-adherence: one was confronted with serious illness of her spouse, one experienced technical problems with the retraining part of the program (which were solved afterwards) and one moved to a new house during the intervention. Two participants who had a (very) low adherence to the program reported that they were too busy with other activities and had other priorities. On the other hand, other patients also experienced interfering circumstances, but were still able to adhere to the intervention. One low-grade glioma patient reported that following the program was burdensome in combination with adjuvant tumor treatment. 


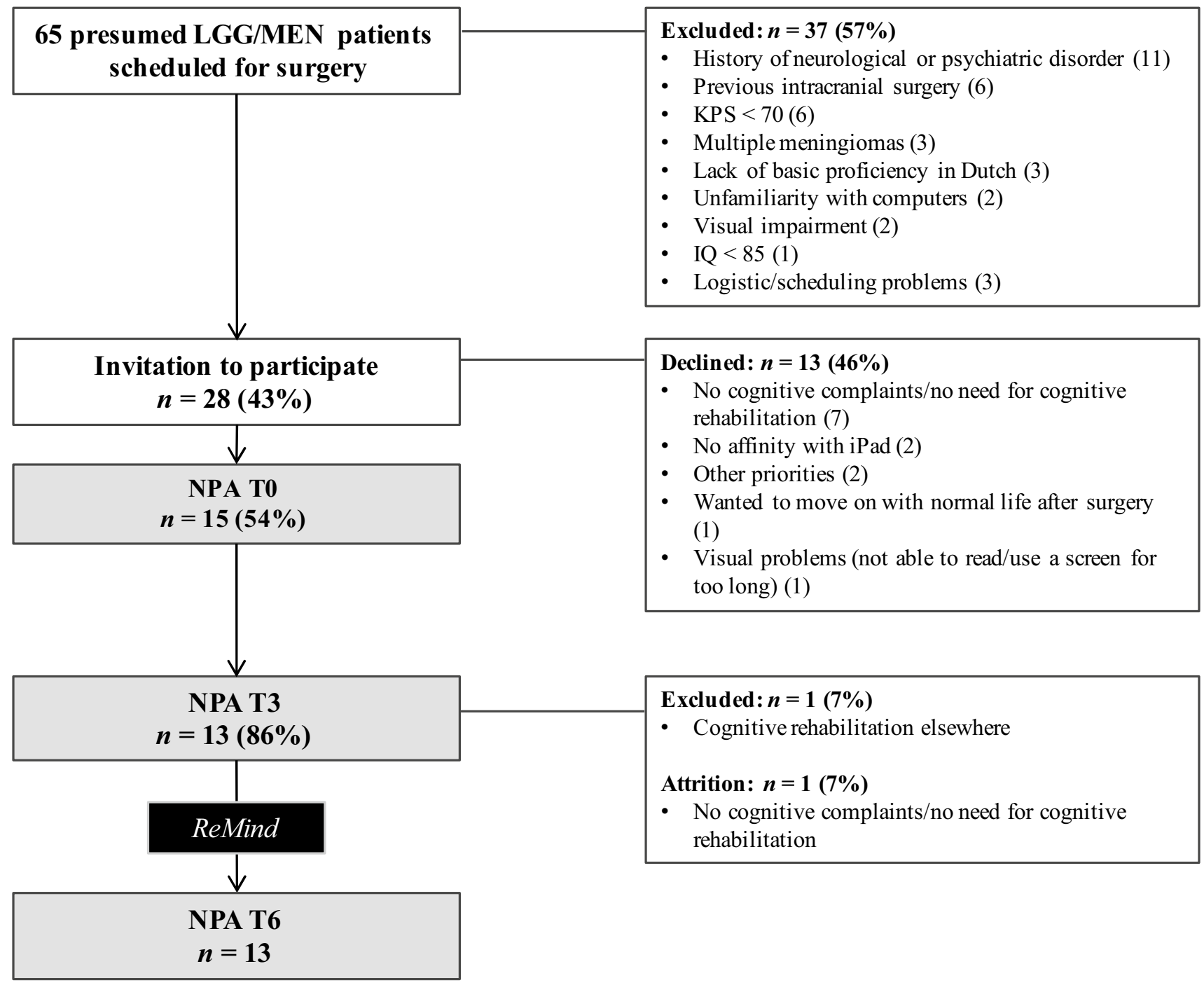

Fig. 2 Flowchart of enrolment and attrition. NPA neuropsychological assessment, $L G G$ low-grade glioma, MEN meningioma, KPS Karnofsky performance score

\section{Patient experience}

Results of the study-specific evaluation questionnaire are listed in Table 2 . The majority evaluated the difficulty and the quantity of psychoeducation, fill-in exercises (to practice with learned strategies) and retraining tasks as sufficient. However, four participants reported that there were too many fill-in exercises included in the strategy training, whereas four other participants rated the retraining as (a bit too) easy and found there were (too) few retraining exercises included in the program. Furthermore, eight patients enjoyed working with ReMind. Using an iPad-app for cognitive rehabilitation was appreciated. Overall, 11 patients (85\%) evaluated the cognitive rehabilitation program ReMind as "good" or "excellent". All participants indicated that they would recommend the program to other brain tumor patients.

\section{Feasibility of neuropsychological assessments}

Three patients were excluded from the study beforehand, because they did not undergo the first neuropsychological assessment (T0) due to (logistical) problems with planning. All 13 participants fully completed neuropsychological assessments, one questionnaire of a participant was not returned.

\section{Discussion}

ReMind is the first cognitive telerehabilitation program specifically developed for adult patients with primary brain tumors. The current pilot study was designed to test the feasibility of an evidence-based telerehabilitation program in 


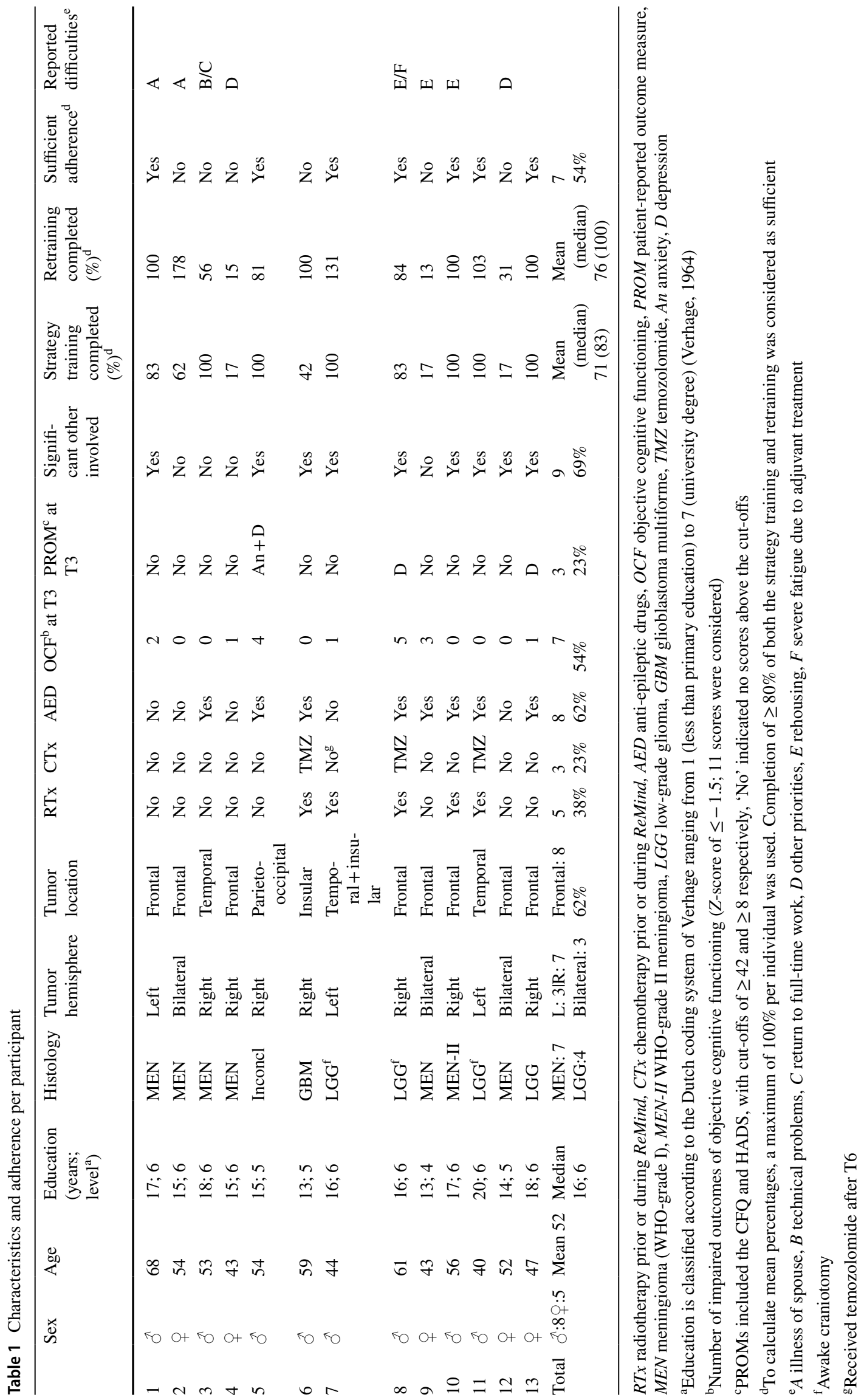


Table 2 Post-intervention ratings of different aspects of ReMind $(n=13)$

\begin{tabular}{|c|c|c|c|}
\hline Difficulty of & (Too) easy & Just right & (Too) difficult \\
\hline Information in strategy training & 3 & 10 & - \\
\hline Fill-in exercises in strategy training & 1 & 10 & 2 \\
\hline Retraining ( $C$-Car game) & 4 & 9 & - \\
\hline Amount/number of & (Too) little/few & About right & (Too) much/many \\
\hline Information in strategy training & 1 & 12 & - \\
\hline Fill-in exercises in strategy training & - & 9 & 4 \\
\hline Retraining exercises ( $C$-Car game) & 4 & 8 & 1 \\
\hline Supervision of the researcher/trainer & - & 13 & - \\
\hline Usefulness of & (Very) useful & Neutral & Not useful \\
\hline Information in strategy training & 7 & 6 & - \\
\hline Fill-in exercises in strategy training & 3 & 9 & 1 \\
\hline Retraining exercises ( $C$-Car game) & 11 & 2 & - \\
\hline (Telephone) contact with the researcher/trainer & 13 & - & - \\
\hline \multirow[t]{2}{*}{ Content addressed daily problems } & Fully/largely & Partly & Not \\
\hline & 8 & 4 & 1 \\
\hline \multirow[t]{2}{*}{ Application of learnt (strategies) in daily life } & Often/regularly & Sometimes & Seldom/never \\
\hline & 3 & 5 & 5 \\
\hline \multirow[t]{2}{*}{ Impact of cognitive problems has changed } & Yes, positively & $\mathrm{No}^{\mathrm{a}}$ & Yes, negatively \\
\hline & 6 & 7 & - \\
\hline \multirow[t]{2}{*}{ Coping with cognitive problems has changed } & Improved coping & $\mathrm{No}^{\mathrm{b}}$ & Worsened coping \\
\hline & 5 & 8 & - \\
\hline \multirow[t]{3}{*}{ Pleasantness of working on ReMind } & (Very) pleasant & Neutral & (Very) unpleasant \\
\hline & 8 & 3 & 2 \\
\hline & Excellent/good & Sufficient & Insufficient/poor \\
\hline Using an iPad-app for cognitive rehabilitation & 11 & 1 & 1 \\
\hline Capability of the researcher/trainer & 13 & - & - \\
\hline Contact with the researcher/trainer & 12 & 1 & - \\
\hline \multirow[t]{2}{*}{ Overall rating of the program } & 11 & 1 & 1 \\
\hline & Yes & No & \\
\hline Recommendation to other brain tumor patients & 13 & 0 & \\
\hline
\end{tabular}

${ }^{a}$ No change, there was no impact on daily life (5) or no change, impact remained the same (2)

${ }^{\mathrm{b}}$ Coping is still good (8), or coping is still not $\operatorname{good}(0)$

the clinical (research) setting in preparation for a larger RCT. The results suggest that, for the subset of interested patients who were included in the study based on specific criteria, cognitive rehabilitation by using the ReMind-app was feasible. Overall, participants were satisfied with the program and dropout was low.

The recruitment of participants to the study was the most challenging aspect of this feasibility study. A substantial part $(57 \%)$ of the patients who were undergoing surgery for lowgrade glioma or meningioma were not eligible based on the exclusion criteria. In hindsight, the exclusion criteria appeared to be overly strict, which, in an attempt to reduce bias through controlling patients' characteristics and potential confounders, potentially compromised the generalizability of the results to the target patient population of patients with presumed lowgrade glioma and meningioma [30, 31]. In particular, a large proportion of patients with a history of neurological/psychiatric disorders or previous intracranial surgery were excluded, although cognitive rehabilitation may be relevant for them as well. Based on these experiences, we adapted the inclusion criteria of the RCT. Furthermore, $46 \%$ of eligible patients declined participation, half of them reporting to feel no need to undergo cognitive rehabilitation at this stage $(n=7)$. Two patients specifically declined participation since an iPad-based intervention was not appealing to them.

With respect to adherence, $54 \%$ of the participants met the criterion for sufficient (completion of $\geq 80 \%$ of both the strategy training and the retraining) adherence, which was comparable with other studies that investigated psychological eHealth interventions in other patient populations [32], but not as high as the adherence to the face-to-face program in our previous RCT $[9,12]$. Whereas our previous study 
included a sample of patients with clinically stable lowergrade gliomas with a disease duration of several years, the patients in the present study participated only shortly after, or even during, the tumor treatment phase of their disease. For example, five patients received adjuvant treatment during the intervention. The patients in our pilot study lived through a turbulent period, in which psychosocial developments, in addition to medical treatment and recovery, are predominating. Some of them resume their work and/ or family care during this period. It may be that, for some patients, undergoing cognitive rehabilitation in this phase is (too) burdensome. Furthermore, in contrast to our previous study, experiencing subjective cognitive (or objective) dysfunction was not an inclusion criterion. Consequently, not all patients in our sample experienced cognitive dysfunction (yet), which may have led to a lack of motivation to fully adhere to the program for some. Along this line, all three participants who reported psychological complaints showed (more than) sufficient adherence rates.

In order to reduce the well-known problems with adherence in remote interventions and, in particular, to find substitutes for the low amount of (face-to-face) supervision, before the start of the pilot study, we incorporated several features into the program that are known to enhance adherence [32-34]. For example, we provided regular guidance during the intervention, through telephone counseling and by provision of feedback from the program itself. Additionally, the program offers the possibility to patients to involve a significant other in the process, an option that nine patients chose. Seven of these patients showed sufficient adherence rates. Despite the efforts made, adherence rates were suboptimal.

Overall, participants who followed the program reported that an iPad-app was an appropriate mode of delivery of cognitive rehabilitation. In fact, this mode enables many patients with brain tumors to follow a cognitive rehabilitation program at their own homes, which is a great advantage since many patients are not allowed to drive due to epileptic seizures. Another important advantage is that patients can follow the program at their own pace and can spread the material over as many sessions as they want, which could be helpful for (vulnerable and/or) older patients in particular [20]. All patients indicated that they would recommend to program to other brain tumor patients. However, some participants indicated that the retraining was too easy for them. Therefore, we decided to expand the retraining with nine more difficult exercises for use in the RCT and beyond, ensuring that the retraining remains challenging for each individual.

In intervention studies, the timing of the intervention is an important, but difficult issue, wherein a balance is sought between intervening not too early, but also not too late. Research in patients with brain tumors demonstrated that the need for supportive care is very high, especially in the early stage of the disease [14]. We hypothesized that early cognitive rehabilitation may enhance the recovery process and may prevent/minimize the negative impact of cognitive side effects of adjuvant treatment, and we decided to start the intervention soon after physical recovery from the surgery and after completion of radiotherapy. At three months after surgery, the intervention could be easily embedded in the existing logistics of our clinical aftercare, thereby minimizing patient burden. Because of the hypothesized preventative effects at this stage, both patients with and without cognitive complaints/deficits were eligible. We assume that several aspects of our cognitive rehabilitation program, for example psychoeducation about cognitive (dys)functioning in patients with brain tumors, could be helpful for a broad group of patients at an early stage.

We have started a larger trial to evaluate the efficacy of ReMind with respect to cognitive functioning and patientreported outcomes, in which patients are consecutively randomized to an intervention group or waiting-list control group by minimization [35] after the 3 months' assessment. Based on the experiences in the pilot study, exclusion criteria were revised to include a broader group of patients and two participating medical centers were added. A 6-month followup assessment was added to the design and participants are requested to keep records of their time spent on the program using registration forms. In addition, a pilot study in 20 glioma patients with stable disease and cognitive complaints is currently being conducted, using the English version of the ReMind-app, at the University of California, San Francisco (UCSF) (clinicaltrials.gov NCT02783495). Ultimately, if the results of the RCT demonstrate beneficial effects of ReMind at the postoperative stage, this telerehabilitation program may enable many patients with brain tumors to follow a cognitive rehabilitation program at their own pace in their own environment early in the course of the disease.

Acknowledgements We would like to thank Sophie Rijnen, Astrid de Munnik, Lieke van den Heuvel, Febe Slikboer and Eileen Kessel for their contribution to the data collection.

Funding This research was supported by the Dutch organization for health research and innovation (ZonMw) (Grant Number: 842003009). Tilburg University developed the ReMind-app. The development was funded by Health Insurers Innovation Foundation, CbusineZ and ' $t$ Hoofdgerecht without commercial interest.

\section{Compliance with ethical standards}

Conflict of interest The authors declare that there is no conflict of interest.

Open Access This article is distributed under the terms of the Creative Commons Attribution 4.0 International License (http://creativecommons.org/licenses/by/4.0/), which permits unrestricted use, distribution, and reproduction in any medium, provided you give appropriate credit to the original author(s) and the source, provide a link to the Creative Commons license, and indicate if changes were made. 


\section{References}

1. Taphoorn MJB, Klein M (2004) Cognitive deficits in adult patients with brain tumours. Lancet Neurol 3(3):159-168. https:// doi.org/10.1016/S1474-4422(04)00680-5

2. Meskal I, Gehring K, Rutten GJM, Sitskoorn MM (2016) Cognitive functioning in meningioma patients: a systematic review. J Neurooncol 128(2):195-205. https://doi.org/10.1007/ s11060-016-2115-z

3. Aaronson NK, Taphoorn MJB, Heimans JJ, Postma TJ, Gundy CM, Beute GN, Slotman BJ, Klein M (2011) Compromised health-related quality of life in patients with low-grade glioma. J Clin Oncol 29(33):4430-4435. https://doi.org/10.1200/ JCO.2011.35.5750

4. Waagemans ML, Van Nieuwenhuizen D, Dijkstra M, Wumkus M, Dirven CMF, Leenstra S, Reijneveld JC, Klein M, Stalpers LJA (2011) Long-term impact of cognitive deficits and epilepsy on quality of life in patients with low-grade meningiomas. Neurosurgery 69(1):72-78. https://doi.org/10.1227/ NEU.0b013e318212badb

5. Tucha O, Smely C, Preier M, Lange KW (2000) Cognitive deficits before treatment among patients with brain tumors. Neurosurgery $47(2): 324-333$

6. Van Loon EMP, Heijenbrok-Kal MH, van Loon WS, Van den Bent MJ, Vincent AJPE., De Koning I, Ribbers GM (2015) Assessment methods and prevalence of cognitive dysfunction in patients with low-grade glioma: a systematic review. J Rehabil Med 47(6):481-488. https://doi.org/10.2340/16501977-1975

7. Claus EB, Black PM (2006) Survival rates and patterns of care for patients diagnosed with supratentorial low-grade gliomas: data from the SEER program, 1973-2001. Cancer 106(6):13581363. https://doi.org/10.1002/cncr.21733

8. Linsler S, Keller C, Urbschat S, Ketter R, Oertel J (2016) Prognosis of meningiomas in the early 1970 s and today. Clin Neurol Neurosurg 149:98-103. https://doi.org/10.1016/j. clineuro.2016.08.007

9. Gehring K, Sitskoorn MM, Gundy CM, Sikkes SAM, Klein M, Postma TJ, van den Bent MJB, Beute GN, Enting RH, Kappelle AC, Boogerd W, Veninga T, Twijnstra A, Boerman DH, Taphoorn MJ, Aaronson NK (2009) Cognitive rehabilitation in patients with gliomas: a randomized, controlled trial. J Clin Oncol 27(22):3712-3722. https://doi.org/10.1200/ JCO.2008.20.5765

10. Zucchella C, Capone A, Codella V, De Nunzio AM, Vecchione C, Sandrini G, Pace A, Pierelli F, Bartolo M (2013) Cognitive rehabilitation for early post-surgery inpatients affected by primary brain tumor: a randomized, controlled trial. J Neurooncol 114(1):93-100. https://doi.org/10.1007/s11060-013-1153-z

11. Maschio M, Dinapoli L, Fabi A, Giannarelli D, Cantelmi T (2015) Cognitive rehabilitation training in patients with brain tumor-related epilepsy and cognitive deficits: a pilot study. J Neurooncol 125(2):419-426. https://doi.org/10.1007/ s11060-015-1933-8

12. Gehring K, Aaronson NK, Taphoorn MJB, Sitskoorn MM (2011) A description of a cognitive rehabilitation programme evaluated in brain tumour patients with mild to moderate cognitive deficits. Clin Rehabil 25(8):675-692. https://doi. org/10.1177/0269215510395791

13. Pace A, Villani V, Parisi C, Di Felice S, Lamaro M, Falcicchio C, Bonucci A, Pugliese P, di Napoli A, Di Lallo D (2016) Rehabilitation pathways in adult brain tumor patients in the first 12 months of disease. A retrospective analysis of services utilization in 719 patients. Support Care Cancer 24(11):4801-4806. https://doi.org/10.1007/s00520-016-3333-9
14. Langbecker D, Yates P (2016) Primary brain tumor patients' supportive care needs and multidisciplinary rehabilitation, community and psychosocial support services: awareness, referral and utilization. J Neurooncol 127(1):91-102. https://doi. org/10.1007/s11060-015-2013-9

15. Bergquist TF, Thompson K, Gehl C, Munoz Pineda J (2010) Satisfaction ratings after receiving internet-based cognitive rehabilitation in persons with memory impairments after severe acquired arain injury. Telemed J E Health 16(4):417-423. https://doi.org/10.1089/tmj.2009.0118

16. Jelcic N, Agostini M, Meneghello M, Bussè C, Parise S, Galano A, Tonin P, Dam M, Cagnin A (2014) Feasibility and efficacy of cognitive telerehabilitation in early Alzheimer's disease: a pilot study. Clin Interv Aging 9:1605-1611. https://doi.org/10.2147/ CIA.S68145

17. Kesler SR, Lacayo NJ, Jo B (2011) A pilot study of an online cognitive rehabilitation program for executive function skills in children with cancer-related brain injury. Brain Inj 25(1):101112. https://doi.org/10.3109/02699052.2010.536194

18. Bray V, Dhillon HM, Bell ML, Kabourakis M, Fiero MH, Yip D, Boyle F, Price MA, Vardy JL (2016) Evaluation of a webbased cognitive rehabilitation program in cancer survivors reporting cognitive symptoms after chemotherapy. J Clin Oncol 35(2):217-225. https://doi.org/10.1200/JCO.2016.67.8201

19. Brennan DM, Mawson S, Brownsell S (2009) Telerehabilitation: enabling the remote delivery of healthcare, rehabilitation, and self management. Stud Health Technol Inform 145:231-248

20. Gehring K, Aaronson NK, Gundy CM, Taphoorn MJB, Sitskoorn MM (2011) Predictors of neuropsychological improvement following cognitive rehabilitation in patients with gliomas. J Int Neuropsychol Soc 17(2):256-266. https://doi.org/10.1017/ S1355617710001530

21. Gualtieri CT, Johnson LG (2006) Reliability and validity of a computerized neurocognitive test battery, CNS Vital Signs. Arch Clin Neuropsychol 21(7):623-643. https://doi. org/10.1016/j.acn.2006.05.007

22. Rijnen SJM, Meskal I, Emons WHM, Campman CAM, Van der Linden SD, Gehring K, Sitskoorn, MM (2017) Evaluation of normative data of a widely used computerized neuropsychological battery: applicability and effects of sociodemographic variables in a Dutch sample. Assessment. https://doi. org/10.1177/1073191117727346

23. Wechsler D (2005) Wechsler Adult Intelligence Scale, third edition. Nederlandstalige bewerking. Technische handleiding. Harcourt Test Publishers, Amsterdam

24. Schmand B, Groenink SC, Van den Dungen M (2008) Letter fluency: psychometrische eigenschappen en Nederlandse normen. Tijdschr Gerontol Geriatr 39(2):64-74

25. Wechsler D (1997) Wechsler memory scale, third edition. The Psychological Corporation, San Antonio, TX

26. Broadbent DE, Cooper PF, FitzGerald P, Parkes KR (1982) The cognitive failures questionnaire (CFQ) and its correlates. Br J Clin Psychol 21:1-16

27. Ponds R, van Boxtel MPJ, Jolles J (2006) De cognitive failure questionnaire als maat voor subjectief cognitief functioneren. Tijdschr Neuropsychol 1(2):37-45

28. Zigmond AS, Snaith RP (1983) The hospital anxiety and depression scale. Acta Psychiatr Scand 67(6):361-370

29. Spinhoven P, Ormel J, Sloekers PPA, Kempen GIJM., Speckens AEM, Van Hemert AM (1997) A validation study of the Hospital Anxiety And Depression Scale (HADS) in different groups of Dutch subjects. Psychol Med 27(2):363-370

30. Gerber DE, Pruitt SL, Halm EA (2015) Should criteria for inclusion in cancer clinical trials be expanded? J Comp Eff Res 4(4):289-291. https://doi.org/10.2217/cer.15.27 
31. Van Spall HGC, Toren A, Kiss A, Fowler RA (2007) Eligibility criteria of randomized controlled trials published in high-impact general medical journals: a systematic sampling review. JAMA 297(11):1233-1240. https://doi.org/10.1001/jama.297.11.1233

32. Beatty L, Binnion C (2016) A systematic review of predictors of, and reasons for, adherence to online psychological interventions. Int J Behav Med 23(6):776-794. https://doi.org/10.1007/ s12529-016-9556-9

33. Donkin L, Christensen H, Naismith SL, Neal B, Hickie IB, Glozier N (2011) A systematic review of the impact of adherence on the effectiveness of e-therapies. J Med Internet Res 13(3):e52. https://doi.org/10.2196/jmir.1772

34. Kelders SM, Kok RN, Ossebaard HC, Van Gemert-Pijnen JE (2012) Persuasive system design does matter: a systematic review of adherence to web-based interventions. J Med Internet Res 14(6):e152. https://doi.org/10.2196/jmir.2104

35. Scott NW, McPherson GC, Ramsay CR, Campbell MK (2002) The method of minimization for allocation to clinical trials: a review. Control Clin Trials 23(6):662-667 